Ophthalmologica 1972;165:557-566

\title{
Index rerum ad Vol. 165
}

\section{Confecit: C. Loeb-Schürch}

$(\mathrm{V})=$ Report - Vortrag - Communication $(\mathrm{B})=$ Book reviews - Buchbesprechungen - Livres nouveaux

$\mathrm{Ab}$ externo trabeculotomy; trabeculotomy

ab externo as operative treatment of

glaucoma simplex, 509 (V) Ablatio retinae, v. Hyaluronsäure,

Indentation sclérochoroïdienne,

Mégalophtalmie, Prevention Acuité visuelle, v. Indentation

sclérochoroïdienne, Pigmentary

retinopathy, Standardization, Voll-

okklusion Aderhaut, v. Aderhautfluoreszenz,

Tyrosinase, Vogt-Koyanagi Harada Aderhautfluoreszenz; normaler und

gestörter Aspekt der Aderhautfluoreszenz, 309 (V) Adler's physiology of the eye, 78 (B)

Ätzverletzungen; die chirurgische

Behandlung der Ätzverletzungen des

Auges, 556 (B) Affections maculaires; ERG et EOG dans

les affections maculaires dégénératives,

396 (V) Aide-lecture, v. Lesehilfen Alkali-burns, v. Collagenolysis Albinisme; etude ophtalmooto-dermato-

logique de Гalbínisme, 398 (V) Alport; syndrome d'Alport et lenticône.

Considerations anatomo-cliniques,

219 (V) Alport's syndrome. Clinicopathological considerations, 1

Amblyopie, v. Mikrostrabismus,

Vollokklusion Amyloidosis, v. Lattice corneal

dystrophy Angioma, v. Haemangiome, Toxo-

plasmose Angiopathy; traumatic retinal angiopathy

(Purtscher), 453 Antigen, v. Corneal antigens, Immuno-

genic factors Applanationstonometrie; zur Praxis der

Applanationstonometrie an der Spalt-

lampe, 271 (V) Aqueous humor, v. Neuroleptiques,

Tyrosinase Aîrophia retinae pigmentosa atypica,

522 (V) Atrophie optique, v. Angiopathy Augenkomplikationen, v. Angiopathy,

Chloroquinschäden, Neuroleptiques,

Prevention, Pupillary block, Rétino-

pathie chloroquinique Augenmuskeln, v. Myogenic palsies,

Oculomotor paralysis, Ophthalmo-

plegie Autoradiographie, v. 8H-Thymidin 
Bâtonnet, v. Pigmentary retinopathy Behçet's disease, v. Immunosuppression Biochemie, v. Cholinesterases, Immuno-genic factors, Tyrosinase

558

Index rerum ad Vol. 165

Biomikroskopie, v. Spaltlampenmikroskopie Blindness, v. Lesehilfen Book reviews, 78 (B), 424 (B), 556 (B) Bouée de sauvetage; cataracte en bouée de sauvetage, 212 (V) Bruchsche Membran; neue elektronenmikroskopische Befunde an der Bruchschen Membran des menschlichen Auges, 331 (V) Brûlures par alcali, v. Collagenolysis Buchbesprechungen, 78 (B), 424 (B), 556 (B) Buphthalmos, v. Ab externo trabeculotomy, Mégalophtalmie Burns, v. Collagenolysis

Calcium dobesilate, v. Human growth

hormone Calf, v. Immunogenic factors Carotis, v. Ophtalmodynamométrie Cataracta anularis, v. Bouée de sauvetage Cataracte, v. Alport's syndrome, Cataractes congenitales, GlaukomKatarakt-Operation, Mégalophtalmie, Peudophakoi Cataractes congenitales. Resultats opératoires et etudes statistiques sur une période de plus de 25 ans (de 1944 à debut 1971), 208 (V) Cécité, v. Lesehilfen Champ visuel, v. Indentation sclérochoroïdienne, Pigmentary retinopathy Chien, v. Keratitis superficialis Chirurgie, v. Ab externo trabeculotomy, Glaukom-Katarakt-Operation, Hyalu-ronsäure, Indentation sclérochoroï-dienne, Keratoprosthesis, Ohr-Haut-knorpel-Plastik, Prevention, Pseudo-phakoi, Pupillary block, Trabeculotomy Chlamydiaceae, v. Keratitis superficialis Chloramphenicol; hydrophilic contact lenses as a new therapeutic approach for the topical use of chloramphenicol and tetracycline, 62 Chloroquin, v. Rétinopathie chloro-quinique

Chloroquinschäden; zur Brauchbarkeit des Rotlichtproñls und der Dunkel-adaptation bei der Diagnostik von Chloroquinschäden, 252 (V)

Cholinesterases; properties of cholin-esterases of human ocular tissues, 153

Choroidal circulation; fluorescein angio-graphy of the choroidal circulation, 533 (V)

Chymotrypsin, v. Prevention

Cicatrisation; le role de Гépithélium dans la cicatrisation cornéenne, 184 (V)

Circulation, v. Choroidal circulation, Debit sanguin rétinien, Fluorométríe

Collagenase, v. Collagenolysis

Collagenolysis and regeneration in corneal burnings, 137

Color vision, v. Pigmentary retinopathy

Complications oculaires, v. Angiopathy, Chloroquinschäden, Neuroleptiques, Prevention, Pupillary block, Rétinopathie chloroquinique

Computer, v. Ordinateurs, Perimetrie

Cone, v. Pigmentary retinopathy

Congrès, v. Netherlands Ophthalmo-logical Society, Société Suisse d'Oph-talmologie

Contact lens, v. Chloramphenicol, Epikeratoprothese

Convergence, v. Synoptophor

Cornea, v. Chloramphenicol, Cicatrisation, Collagenolysis, Corneal antigens, Epikeratoprothese, Hornhautverände-rungen, Immunogenic factors, Imuran, Keratitis superficialis, Keratokonus, 
Keratoprosthesis, Lattice corneal dystrophy, Macular cornea dystrophy, Migration, Neuroleptiques

Corneal antigens; localisation of corneal antigens, 543 (V)

Corneal graft, v. Immunogenic factors, Imuran

Corps étranger, v. Migration

Corps vitré, v. 3H-Thymidin, Hyaluron-säure, Prevention, Schneckenspuren, Spaltlampenmikroskopie

Cristallin, v. Alport, Alport's syndrome,

Index rerum ad Vol. 165

559

Bouée de sauvetage, Cataractes con-

génitales, Mégalophtalmie, Neuro-

leptiques, Prevention Cyanakrylatadhaesiva, v. Epikerato-

prothese Cysts, v. Pars-plana-Zysten Cytostatics, v. Immunosuppression

Deafness, v. Albinisme, Alport's

syndrome, Vogt-Koyanagi Harada Debit sanguin rétinien; essai de mesure

du debit sanguin rétinien relatif par

l'angiographie fluorescéinique, 303 (V) Décollement rétinien, v. Hyaluronsäure,

Indentation sclérochoroïdienne,

Mégalophtalmie, Prevention Desinfektion, v. Applanationstonometrie Diabetic retinopathy, v.

Human growth

hormone, Hyaluronsäure, Light

coagulation Diagnosis of metabolic eye diseases,

78(B) Diagnostic diftérentiel, v. Myogenic

palsies, Sepsis lenta, Système photo-pique, Toxoplasmose, Vogt-Koyanagi

Harada Diagnostik, v. Chloroquinschäden,

Ophthalmodynamométrie, Ophthalmo-

echographie Diathermic barrage, v. Prevention Diplopia, v. Secondary correction Dog, v.

Keratitis superficialis Doxium, v. Human growth hormone Dunkeladaptation, v.

Chloroquinschäden Durchblutung, v. Choroidal circulation,

Debit sanguin rétinien, Fluorométrie Dynamographie, v. Ophtalmodynamo-

métrie Dynamometrie, v. Ophtalmodynamo-

métrie Dysplasia epiphysalis, with ocular

anomalies, 554 (V) Dystrophie cornéenne, v. Lattice corneal

dystrophy, Macular cornea dystrophy

Echographie, v. Ophthalmoechographie, Ultrasonic diagnostics

EEG, v. Oculomotor paralysis

Effet de photocoagulation; contrôle de $\Gamma$ effet de la photocoagulation par angiographie

fluorescéinique, $316(\mathrm{~V})$

Elektronenmikroskopie, v. Bruchsche Membran, Klumpenzellen, Pars-plana-Zysten

Eîektrophorese, v. Immunogenic factors

Embolie graisseuse, v. Angiopathy

Embryologie, v. 3H-Thymidin, Pars-plana-Zysten

EMG, v. Myogenic palsies, Ophíhalmo-plegie

Endovascular haemangioma, v. Haem-angiome 
Enzephalitis, v. Vogt-Koyanagi Harada

EOG, v. Affections maculaires, Pigmentary retinopathy, Rétinopathie chloro-quinique,

Rétinopathie pigmentaire, Système photopique

Epikeratoprothese; zur Anwendung sogenannter Epikeratoprothesen, 343 (V)

Epithel, v. Cicatrisation

Epithelium pigmentaire, v. Choroidal circulation, Rétinopathie pigmentaire

ERG, v. Affections maculaires, Albinisme, Pigmentary retinopathy, Rétinopathie

chloroquinique, Rétinopathie pigmentaire, Système photopique

Exophthalmos, v. Phlebography

Experimentelle Forschung, v. Cholin-esterases, Collagenolysis, Immunogenic factors,

Kälteapplikation

Extraction du cristallin, v. Prevention

Farbsinn, v. Pigmentary retinopathy

Fettembolie, v. Angiopathy

Fixation excentrique, v. Mikrostrabismus,

Vollokklusion Fixierlampen, v. Applanationstonometrie Fluorescein angiographie studies of the peripapillary and perilimbal regions

in simple, capsular and low-tension

glaucoma, 424 (B) Fluoroangiographie, v. Aderhautfluores-

zenz, Choroidal circulation, Debit

560

Index rerum ad Vol. 165

sanguin rétinien, Effet de photo-coagulation, Optic disc, Pigmentary retinopathy, Retinopathia serosa centralis, Rétinopathie chloroquinique

Fluorometrie; direkte fluorometrische Untersuchungen am Augenhintergrund, 297 (V)

Functions visuelles, v. Indentation scléro-choroïdienne, Pigmentary retinopathy, Sliding-door

effect

Foreign body, v. Migration

Formalin, v. Epikeratoprothese

Fremdkörper, v. Migration

Fundus flavimaculatus. Resume, 390 (V)

Fusion, v. Synoptophor

Gefässe, v. Aderhautfluoreszenz, Angiopathy, Choroidal circulation, Debit sanguin rétinien, Epikeratoprothese, Fluorometrie, Haemangiome, Human growth hormone, Imuran, Inflamed eyes, Light coagulation, Masern, Modellbetrachtungen, Ophthalmo-dynamométríe, Optic disc, Pars-plana-Zysten, Toxoplasmose

Gel hydrophile, v. Chloramphenicol

Genetics, v. Albinisme, Alport's syndrome, Atrophia retinae, Fundus flavimaculatus,

Keratokonus, Macular cornea dystrophy, Mégalophtalmie, Mikrostrabismus, Pigmentary

retinopathy, Système photopique

Gesichtsfeld, v. Indentation scléro-choroïdienne, Pigmentary retinopathy

Glaskörper, v. 3H-Thymidin, Hyaluron-säure, Prevention, Schneckenspuren,

Spaltlampenmikroskopie

Glaucoma, v. Ab externo trabeculotomy, Glaukom-Katarakt-Operation, Hornhautveränderungen, Lattice corneal dystrophy, Pupillary block, Trabeculotomy 
Glaucome congenital incomplet, v. Mégalophtalmie

Glaukom-Katarakt-Operation; kombi-nierte Glaukom-Katarakt-Operation, 203 (V)

Glia, v. Optic disc

Glomerulonephritis, v. Alport's syndrome

Glycostéroides, v. Lattice corneal

dystrophy Greffe cornéenne, v. Immunogenic

factors, Imuran

Haemangiome; endovasale Haemangiome der Vena angularis, 472

Haemorrhagie, v. Human growth hormone, Sepsis lenta

Harada's disease, v. Vogt-Koyanagi Harada

Hemeralopia, v. Pigmentary retinopathy

Hérédité, v. Albinisme, Alport's

syndrome, Atrophia retinae, Fundus flavimaculatus, Keratokonus, Macular cornea dystrophy,

Mégalophtalmie, Mikrostrabismus, Pigmentary retinopathy, Système photopique

Histochemie, v. Collagenolysis, Lattice corneal dystrophy

Histologie, v. Alport's syndrome, Boué $\varepsilon$ de sauvetage, Collagenolysis, Haemangiome, Keratitis

superficialis, Klum-penzellen, Lattice corneal dystrophy, Macular cornea dystrophy,

Schneckenspuren, Sepsis lenta

Hormon, v. Human growth hormone

Hornhaut, v. Chloramphenicol, Cicatrisation, Collagenolysis, Corneal antigens,

Epikeratoprothese, Hornhautveränderungen, Immunogenic factors, Imuran, Keratitis

superficialis, Keratokonus, Keratoprosthesis, Lattice corneal dystrophy, Macular cornea

dystrophy, Migration, Neuroleptiques

Hornhautveränderungen beim chronischen Glaukom, 438

3H-Thymidin; autoradiographische Untersuchungen mit 3H-Thymidin zur Ent-wicklung der

Glaskörperrindenzellen, 175 (V)

Human growth hormone; the correlation between human growth hormone (HGH) concentration in blood plasma and the evolution of diabetic retinopathy, 71

Index reram ad Vol. 165

561

Humeur aqueuse, v. Neuroleptiques,

Tyrosinase Hund, v. Keratitis superficialis Hyaline Degeneration, v. Hornhaut-

veränderungen Hyaluronsäure als Glaskörpersubstituent,

351 (V) Hydrophilic gel, v. Chloramphenicol Hyperlipidämie; Risikofaktor Hyper-

lipidämie, 424 (B) Hypophyse, v. Human growth hormone

Immunogenic factors in corneal stroma, $523(\mathrm{~V})$

Immunologie, v. Immunogenic factors, Imuran, Inflamed eyes

Immunosuppression; indications de Гimmunosuppression par cytostatiques en ophtalmologie,

$176(\mathrm{~V})$

Immuno-suppression, v. Imuran

imuran; the treatment of corneal transplantations with imuran, 529 (V)

Indentation sclérochoroïdienne; le décol-lement de la rétine opéré par les techniques

d'indentation scléro-choroïdienne. Résultats fonctionnels, 417 (V)

Infektion, v. Keratitis superficialis, Masern 
Inflamed eyes; observations on inflamed eyes after influencing the blood supply, with possible relationship to immuno-logical processes, 545 (V)

Instrument, v. Lesehilfen, Ophthalmo-echographie, Synoptophor

Iridektomie, v. Pupillary block

Iris, v. Klumpenzellen, Pupillary block

Jumeaux, v. Keratokonus

Kälteapplikation; Temperaturmessungen an der Netzhaut während Kälte-applikationen, 284 (V)

Kalb, v. Immunogenic factors

Kammerwasser, v. Neuroleptiques, Tyrosinase

Kaninchen. v. 3H-Thymidin

Katarakt, v. Alport's syndrome, Bouée de sauvetage, Cataractes congénitales. Glaukom-

Katarakt-Operation, Mégalophtalmie, Pseudophakoi

Keratektomie, v. Keratitis superficialis

Keratitis superficialis; zur Therapie der Keratitis superficialis chronica (Ueber-reiter) des

Deutschen Schäferhundes, 187 (V)

Keratokonus; konkordanter Keratokonus bei eineiigen Zwillingen, 449

Keratoplastik; der Einfluss der Naht-technik auf den Verlauf und das End-resultat bei der perforierenden Keratoplastik, 291 (V)

Keratoprosthesis. Development and indications, 540 (V)

Klumpenzellen; die Koganeisdien Klumpenzellen. Eine klinische, histo-logische und elektronenmikroskopische Studie, 198 (V)

Koganeische Klumpenzellen, v. Klumpenzellen

Kongresse, v. Netherlands Ophthalmo-logical Society, Schweizerische Ophthal-mologische

Gesellschaft

Kontaktlinse, v. Chloramphenicol, Epikeratoprothese

Konvergenz, v. Synoptophor

Korrespondenz; Qualifikation und Klassifikation der binokularen Korrespondenz, 241 (V)

Kryode, v. Kälteapplikation

Kystes, v. Pars-plana-Zysten

Lähmung, v. Myogenic palsies, Oculomotor paralysis, Ophthalmoplegie

Lapin, v. 3H-Thymidin

Lattice corneal dystrophy; comparative histopathological and clinical findings in eyes with

lattice corneal dystrophy of two different types, 15

Lens, v. Alport, Alport's syndrome, Bouée de sauvetage, Cataractes congénitales.

Mégalophtalmie, Neuroleptiques, Prevention

Lenticonus, v. Alport, Alport's syndrome

562

Index rerum ad Vol. 165

Lentille de contact, v. Chloramphenicol,

Epikeratoprothese Lesehilfen; elektronische Orieníierungs-

und Lesehilfen für den Blinden, 166 (V) Lichtfrequenzwandler, v. Lesehilfen Light coagulation treatment of diabetic

retinopathy in the Eye Hospital,

Rotterdam, 548 (V) Linse, v. Alport, Alport's syndrome,

Bouée de sauvetage, Cataractes 
congénitales, Mégalophtalmie, Neuro-

leptiques, Prevention Livres nouveaux, 78 (B), 424 (B), 556 (B) Luxatio lentis, v.

Mégalophtalmie

Macula, v. Affections maculaires, Pigmentary retinopathy, Rétinopathie chloroquinique

Macular cornea dystrophy; problems round hereditary macular dystrophy of the cornea

(Groenouw type II), 551 (V)

Makrophagen, v. Klumpenzellen

Malformations, v. Alport's syndrome

Masern; zum klinischen Bild und Verlauf der Rétinopathie bei Masern, 332 (V)

Measles, v. Masern

Medikament, v. Applanationstonometrie, Chloramphenicol, Human growth hormone,

Immunosuppression, Imuran

Megalocornea, v. Mégalophtalmie

Mégalophtalmie, subluxation cristal-linienne et cataracte; décollement rétinien, 409 (V)

Melanoblastoma, v. Tyrosinase

Melanozyten, v. Albinisme

Meningoenzephalitis, v. Vogt-Koyanagi Harada

Methode, v. Applanationstonometrie, Chloroquinschäden, Fluorometrie, Glaukom-Katarakt-

Operation, Hyaluronsäure, Indentation scléro-choroïdienne, Keratoplastik, Motion attachment,

Ohr-Hautknorpel-Plastik, Ophtalmodynamométrie, Ophthalmo-echographie, Prevention,

Pseudophakoi, Trabeculotomy

Microaneurysma, v. Human growth

hormone Microscopie électronique, v. Bruchsche

Membran, Klumpenzellen, Pars-plana-

Zysten Migration; Beobachtungen über die

Migration von kleinsten intrakornealen

Fremdkörpern, 195 (V) Mikrostrabismus, 236 (V) Missbildungen, v. Alport's syndrome

Modellbetrachtungen zum Verschluss

pathologischer Netzhautgefässe durch

Photokoagulation, 164 (V) Motilität, v. Myogenic palsies, Ophthal-

moplegie Motion attachment; forced motion

attachment for the Goldmann

perimeter, 482 Mucopoîysaccharidosis, v. Macular

cornea dystrophy Muscles oculaires, v. Myogenic palsies,

Oculomotor paralysis, Ophthalmo-

plegie Myogenic palsies; the diagnosis of

myogenic ocular-motor palsies, 513 (V) Myopie, v. Prevention

Nahttechnik, v. Keratoplastik Narbenbildung, v. Cicatrisation Nephropathie, v. Alport's

syndrome Nerf optique, v. Optic disc Netherlands Ophthalmological Society, 163rd Meeting, Nijmegen 1969, 490-555 (V) Netzhaut, v. Affections maculaires, Angiopathy, Atrophia retinae, Chloroquinschäden, Choroidal circulation, Human growth hormone, Hyaluronsäure,

Kälteapplikation, Light coagulation, Masern, Modellbetrachtungen, Pars-plana-Zysten, Pigmentary retinopathy, Prevention, Retinopathia serosa centralis, Rétinopathie chloroquinique, Rétinopathie pigmentaire, Schneckenspuren, Sepsis lenta, Sliding-door effect, Spaltlampenmikroskopie, Toxoplasmose

Index rerum ad Vol. 165 
Neuroleptiques; etude de Thumeur aqueuse dans les traitements prolongés aux neuroleptiques, $200(\mathrm{~V})$

Neuro-Ophthalmologie, v. Ophthalmo-plegie

Nystagmus, v. Ophthalmoplegie

Oculomotor paralysis; cyclic oculomotor paralysis, with cyclic changes in the EEG, 497 (V)

$£$ Edème papillaire, v. Vogt-Koyanagi Harada

Ohr-Hautknorpel-Plastik bei Lidrand-tumoren, 464

Operation, v. Ab externo trabeculotomy, Glaukom-Katarakt-Operation, Hyaluronsäure, Indentation scléro-choroïdienne, Keratoprosthesis, Ohr-Hautknorpel-Plastik, Prevention, Pseudophakoi, Pupillary block, Trabeculotomy

Ophtalmie sympathique, v. Immuno-suppression

Ophtalmodynamométrie et ophtalmo-dynamographie, méthodes complé-mentaires dans le diagnostic des stenoses ou thromboses carotidiennes, 258 (V)

Ophthalmoechographie; Fortschritte in der Ophthalmoechographie mit einem neuen

Diagnostikgerät, 245 (V)

Ophthalmoplegie; internukleäre Ophthalmoplegie, 320 (V)

Optic disc; colour and fluorescence of the optic disc, 100

Opticusatrophie, v. Angiopathy

Ordinateur, v. Perimetrie

Ordinateurs; Гutilisation des ordinateurs en ophtalmologie, 267 (V)

Orientierungshilfen, v. Lesehilfen

Osteodysplasie; über Augensymptome bei fibröser Osteodysplasie, 270 (V)

Paedo-Ophthalmologie, v. Cataractes congénitales, Dysplasia epiphysalis, Vollokklusion

Pannus glaucomatosus, v. Hornhaut-veränderungen

Papule, v. Optic disc, Vogt-Koyanagi Harada

Papillenoedem, v. Vogt-Koyanagi Harada

Paralysie, v. Myogenic palsies, Oculomotor paralysis, Ophthalmoplegie

Pars-plana-Zysten; elektronenmikrosko-pische Beobachtungen an Pars-plana-Zysten, 327 (V)

Penicillamine, v. Collagenolysis

Perimetrie, v. Motion attachment

Perimetrie, Automatisierung und Informationstheorie, 173 (V)

Phlebography; angular-vein phlebography as an aid to the diagnosis of intra-orbital processes, $505(\mathrm{~V})$

Photocoaguiation, v. Effet de photo-coagulation, Light coagulation, Modell-betrachtungen,

Prevention, Retino-pathia serosa centralis

Photopisches System, v. Système photopique

Piébaldisme, v. Albinisme

Pigmentary retínopathy; visual functions in pericentral and central pigmentary retinopathy, 38

Pigmentepithel, v. Choroidal circulation, Rétinopathie pigmentaire

Pituitary gland, v. Human growth hormone

Plastic surgery, v. Ohr-Hautknorpel-Plastik, Secondary correction

Poliosis, v. Vogt-Koyanagi Harada

Pression oculaire, v. Ab externo trabeculotomy, Pupillary block 
Prevention of retinal detachment by a circumferential barrage prior to lens extraction in highmyopic eyes, 125

Prognose, v. Human growth hormone

Prolin, v. Cicatrisation

Proteins, v. Immunogenic factors

Pseudophakoi; Binkhorst's method of implantation of pseudophakoi in unilateral traumatic cataract, $490(\mathrm{~V})$

564

Index rerum ad Vol. 165

Pupillary block and angle-closure

glaucoma, 109 Purtscher's disease, v. Angiopathy

Quellpunkt, v. Retinopathia serosa centralis

Rabbit, v. 3H-Thymidin

Radiographie, v. Phlebography

Reading-aids, v. Lesehilfen

Recherches expérimentales, v. Cholin-esterases, Collagenolysis, Immunogenic factors,

Kälteapplikation

Refraction, v. Prevention, Sliding-door effect

Regeneration corneenne, v. Collagenolysis

Retina, v. Affections maculaires, Angiopathy, Atrophia retinae, Chloroquin-schäden, Choroidal circulation, Human growth hormone, Hyaluronsäure, Kälteapplikation, Light coagulation, Masern, Modellbetrachtungen, Pars-plana-Zysten, Pigmentary retinopathy, Prevention, Retinopathia serosa centralis, Rétinopathie chloroquinique, Rétinopathie pigmentaire, Schnecken-spuren, Sepsis lenta, Sliding-door effect, Spaltlampenmikroskopie, Toxo-plasmose Retinal detachment, v. Hyaluronsäure, Indentation sclérochoroïdienne, Mégalophtalmie, Prevention

Retinopathia serosa centralis; Licht-koagulation der Retinopathia serosa centralis, 366 (V)

Rétinopathie, v. Masern

Rétinopathie chloroquinique, 81

Rétinopathie diabétique, v. Human growth hormone, Hyaluronsäure, Light coagulation

Rétinopathie pigmentaire; La rétinopathie pigmentaire unilatérale existe-t-elle? A propos de deux nouvelles observations, $402(\mathrm{~V})$

Rétinopathie pigmentaire, v. Pigmentary retinopathy

Retinoscopy, v. Sliding-door effect

Rieger's dysplasia, v. Dysplasia

epiphysalis Rod, v. Pigmentary retinopathy Rotlichtprofil, v. Chloroquinschäden Rougeole, v.

Masern

Scar-formation, v. Cicatrisation

Scherenphänomen, v. Sliding-door effect

Schielen, v. Mikrostrabismus, Synop-tophor, Vollokklusion

Schneckenspuren; zur Kenntnis von Substrat und Bedeutung der sogenann-ten Schneckenspuren der Retina, 360 (V)

Schweizerische Ophthalmologische Gesellschaft, 64. Generalversammlung, Locarno 1971, 164$423(\mathrm{~V})$

Secondary correction of post-traumatic changes in the orbit with alloplastic material, 519 (V) 
Sehschärfe, v. Indentation scléro-

choroïdienne, Pigmentary retinopathy, Standardization, Vollokklusion

Sepsis lenta; hémorragies rétiniennes et cérébrales dans un cas de sepsis lenta. Correlation anatomo-clinique, $373(\mathrm{~V})$

Sight-restoration after long-term blindness, 78 (B)

Skiaskopie, v. Sliding-door effect

Skotom, v. Pigmentary retinopathy

Sliding-door effect; the double sliding-door effect, 117

Société Suisse d'Ophtalmologie, 64c Assembléé générale, Locarno 1971, 164-423 (V)

Spaltlampenmikroskopie; zur Spaltlampenmikroskopie des Glaskörpers, 277 (V)

Squint, v. Mikrostrabismus, Synoptophor, Vollokklusion

Stäbchen, v. Pigmentary retinopathy

Standardization; the standardization of visual-acuity tests for medical examinations and reports, $495(\mathrm{~V})$

Stenose, v. Ophtalmodynamométrie

Steroid-Glykoside, v. Lattice corneal dystrophy

Index rerum ad Vol. 165

565

Strabisme, v. Mikrostrabismus, Synop-

tophor, Vollokklusion Surdité, v. Albinisme, Alport's syndrome,

Vogt-Koyanagi Harada Surgery, v. Ab externo trabeculotomy,

Glaukom-Katarakt-Operation,

Hyaluronsäure, Indentation scléro-

choroïdienne, Keratoprosthesis, Ohr-

Hautknorpel-Plastik, Prevention,

Pseudophakoi, Pupillary block,

Trabeculotomy Sympathische Ophthalmie, v. Immuno-

suppression Syndrome, v. Alport, Alport's syndrome,

Vogt-Koyanagi Harada Synoptophor; erste Erfahrungen mit

einem modifizierten Synoptophor,

221 (V) Système photopique; dégénérescence

progressive du système photopique,

$392(\mathrm{~V})$

Taubheit, v. Albinisme, Alport's syndrome, Vogt-Koyanagi Harada

Temperaturmessungen, v. Kälte-applikation

Tension oculaire, v. Ab externo trabeculotomy, Pupillary block

Test, v. Standardization

Tetracycline, v. Chloramphenicol

Therapie, v. Chloramphenicol, Collagenolysis, Epikeratoprothese, Glaukom-Katarakt-Operation, Human growth hormone, Hyaluronsäure, Immuno-suppression, Imuran, Inflamed eyes, Keratitis superficialis, Light coagulation, Neuroleptiques, Trabeculotomy

Thrombose, v. Ophtalmodynamométrie

Tonometrie, v. Applanationstonometrie

Toxoplasmose; angiome rétinien et toxo-plasmose, $384(\mathrm{~V})$

Trabeculotomie, v. Ab externo trabeculotomy 
Trabeculotomy, $502(\mathrm{~V})$

Transplantat, v. Immunogenic factors, Imuran

Transplantation, v. Keratoprosthesis

Trauma, v. Angiopathy, Secondary

correction Tumor, v. Haemangiome, Ohr-Haut-

knorpel-Plastik, Tyrosinase Twins, v. Keratokonus Tyrosinase activity of aqueous humour

in uveal melanoblastoma, 459 Tyrosinase, v. Albinisme

Ueberreiter'sche Keratitis, v. Keratitis superficialis

Ultrasonic diagnostics in ophthalmology, 517 (V)

Uvea, v. Aderhautfluoreszenz, Tyrosinase, Vogt-Koyanagi Harada

Uveitis, v. Immunosuppression, Vogt-Koyanagi Harada

Vaisscaux, v. Aderhautfluoreszenz, Angiopathy, Choroidal circulation, Debit sanguin rétinien, Epikeratoprothese, Fluorometrie, Haemangiome, Human growth hormone, Imuran, Inflamed eyes, Light coagulation, Masern, Modellbetrachtungen, Ophthalmodynamométrie, Optic disc, Pars-plana-Zysten, Toxoplasmose

Varia, 79

Veau, v. Immunogenic factors

Verätzungen, v. Collagenolysis

Vererbung, v. Albinisme, Alport's syndrome, Atrophia retinae, Fundus flavimaculatus,

Keratokonus, Macular cornea dystrophy, Mégalophtalmie, Mikrostrabismus, Pigmentary

retinopathy, Système photopique

Vernarbung, v. Cicatrisation

Vessels, v. Aderhautfluoreszenz, Angiopathy, Choroidal circulation, Debit sanguin rétinien, Epikeratoprothese, Fluorometrie, Haemangiome, Human growth hormone, Imuran, Inflamed eyes, Light coagulation, Masern, Modellbetrachtungen, Ophtalmodynamométrie, Optic disc, Pars-plana-Zysten, Toxoplasmose

566

Index rerum ad Vol. 165

Virus, v. Vogt-Koyanagi Harada

Visual acuity, v. Indentation scléro-choroïdienne, Pigmentary retinopathy, Standardization, Vollokklusion

Visual field, v. Indentation scléro-choroïdienne, Pigmentary retinopathy

Visual functions, v. Indentation scléro-choroïdienne, Pigmentary retinopathy, Sliding-door effect

Vision de la couleur, v. Pigmentary retinopathy

Vitiligo, v. Vogt-Koyanagi Harada

Vitreous body, v. 3H-Thymidm, Hyaluronsäure, Prevention, Schneckenspuren, Spaltlampen-

mikroskopie

Vogt-Koyanagi Harada; particular aspects of the Vogt-Koyanagi Harada syndrome, 425

Vollokklusion; faziale Vollokklusion des führenden Auges bei grösseren Kindern mit

Schielamblyopie und exzentrischer Fixation, 230 (V)

Wachstumshormon, v. Human growth hormone

Z. Zapfen

v. Pigmentary retinopathy Zirkulation

v. Choroidal circulation,

Debit sanguin rétinien 
Fluorométrie Zwillinge

v. Keratokonus Zysten

v. Pars-plana-Zysten Zytostatika

v. Immunosuppression 